\title{
Linx
}

Revue des linguistes de l'université Paris X Nanterre

9 | 1997

Émile Benveniste. Vingt ans après

\section{Benveniste et la représentation de la « discipline linguistique »}

\section{Christian Puech}

\section{(2) OpenEdition}

\section{Journals}

Édition électronique

URL : http://journals.openedition.org/linx/1090

DOI : 10.4000/linx.1090

ISSN : 2118-9692

Éditeur

Presses universitaires de Paris Nanterre

\section{Édition imprimée}

Date de publication : 1 avril 1997

Pagination : 385-396

ISSN : 0246-8743

\section{Référence électronique}

Christian Puech, «Benveniste et la représentation de la « discipline linguistique » », Linx [En ligne],

9 | 1997, mis en ligne le 25 juillet 2012, consulté le 19 avril 2019. URL : http://journals.openedition.org/ $\operatorname{linx} / 1090 ; \mathrm{DOI}:$ 10.4000/linx.1090 


\title{
Benveniste et la représentation de la "discipline linguistique"
}

\author{
Christian Puech
}

\begin{abstract}
$\mathrm{O}$ n trouve chez E. Benveniste, comme chez la plupart des linguistes fondateurs $\mathrm{du}$ siècle, les éléments d'une représentation de la linguistique. Nous désignons par là non la conception de la science qui guide leurs travaux, c'est à dire l'ensemble des considérations qui concernent l'objet, les procédures, la méthode, les "niveaux de l'analyse linguistique ", mais plutôt le cadre de valeurs en apparence périphériques dans lequel cette conception de la science permet de se situer, de s'orienter et surtout de se transmettre, sur un plan qui n'est donc plus strictement cognitif, mais culturel et disciplinaire.

Le point de vue disciplinaire ${ }^{1}$ que j'essaierai ici de circonscrire, double l'effort scientifique proprement dit, mais s'en distingue aussi par sa perspective propre : reformulations, contextualisation des connaissances, mises en relations multiples, paradigmatisation... Quand Saussure introduit par exemple une distinction entre matière et objet de la linguistique, il s'agit pour lui de délimiter sans doute « l'objet intégral » de la linguistique, mais il s'agit aussi de situer, contextualiser cet objet en l'inscrivant dans une configuration disciplinaire où la linguistique coexiste et s'articule à d'autres points de vue, s'inscrit dans une temporalité.

Plus largement, et dans ce qu'on peut considérer comme la descendance saussurienne, quand les linguistes proclament des filiations et des affiliations (institutionnelles, linguistiques, nationales, culturelles, épistémologiques... Chiss et Puech, 1995), quand ils se réclament d'un précurseur, ils organisent un champ de savoir homogène, par un certain agencement de la mémoire disciplinaire (Chiss et Puech 1994).
\end{abstract}

\footnotetext{
${ }^{1}$ Cf. pour une analyse plus développée, Chiss et Puech 1995 .
} 


\section{Christian Puech}

Quand ils définissent pour la linguistique un objet propre - Benveniste ne manque jamais d'étendre la recommandation saussurienne qui concerne chez Saussure la sémiologie aux principes premiers de la linguistique : celle-ci doit d'abord se définir elle-même - il s'agit pour eux d'indexer, identifier, décrire les principaux domaines de la discipline où l'objet trouve sa place légitime à côté d'autres objets. Il s'agit en somme - et on peut peut-être voir là dans une certaine mesure une part essentielle de l'héritage saussurien - de faire jouer une dialectique des « résultats et des points de vue $»^{2}$.

Enfin, fixer des tâches programmatiques, c'est compléter par la dimension projective la cohérence synchronique et rétrospective de la discipline. De ce point de vue, la sémiologie est, dans la linguistique d'inspiration structurale, au moins autant un domaine à explorer qu'un horizon à ouvrir, à définir pour se situer. On peut ainsi considérer que la distinction proposée par Benveniste entre sémiotique et sémantique s'inscrit ici par exemple dans une «série " - à laquelle elle est sans doute irréductible - déjà longue de ces propositions théoriques «projectives » qui ouvrent une perspective en situant la linguistique dans une constellation disciplinaire dont elle hérite et qu'elle modifie. Bref, le point de vue disciplinaire serait le point de vue d'une représentation $d u$ savoir sous trois aspects principaux : rétrospection, délimitation du champ et projection.

On ne trouve bien sûr jamais dans les textes généraux des linguistes les plus prestigieux depuis les années 20 ces modalités d'une conscience disciplinaire à l'état pur ${ }^{3}$. Mais elles sont toujours impliquées, à un moment ou à un autre dans leurs exposés synthétiques et généraux selon des proportions et modalités variées. Du «traité de linguistique générale » aux « introductions », «prolégomènes », « essais », comptes-rendus..., le discours de spécialité n'ignore jamais complètement la nécessité exotérique de se situer pour se transmettre.

De ce point de vue, d'ailleurs, la question des titres des ouvrages de linguistique générale évoquée les jours précédents n'est ni totalement triviale ni particulièrement futile dans la perspective disciplinaire que nous nous efforçons d'adopter ici. Entre 1921 et 1923, trois ouvrages fondamentaux paraissent, on le sait, sous le même titre: "Le langage» (J. Vendryes, O. Jespersen, E. Sapir). L'entrée disciplinaire est ici clairement assumée : c'est celle de l'objet, et non celle de la théorie dont il relève. Ce qui ne signifie pas pour autant que les ouvrages annoncés par ce titre ignorent cette dernière perspective. Le Livre I du Langage d'O. Jespersen est au contraire entièrement occupé par une histoire des théories linguistiques et de ses problématiques depuis l'Antiquité. Mais cette histoire n'a en fait pour fonction que celle « $\mathrm{d}^{\prime}$ introduction » aux questions essentielles qu'il faudrait affronter. Ce privilège

\footnotetext{
2 Il existe peut-être en linguistique, explique à peu près Hjelmslev, des "résultats définitifs », il n'existe pas de "points de vue » définitifs (Le langage, 1966, p. 27). R. Engler croit pouvoir assigner une origine à ce topos de la linguistique moderne dans le débat entre Ascoli et Meyer au début du siècle.

${ }^{3}$ Cf. Chiss et Puech 1994 et 1995.
} 
accordé à l'objet, on le retrouve bien dans la recension de L. Bloomfield en 1927 («On Recent Works in General Linguistics», Modern Philology 25) qui rassemble des ouvrages de linguistique générale depuis 1970 sous un critère homogène, défini de la manière suivante :

Au delà du désir qu'ils manifestent de "mettre au point une méthode scientifique pour rendre compte $d u$ changement linguistique.... ces ouvrages expriment l'ambition "d'assigner au langage sa place dans l'univers ».

Ne peut-on penser qu'au regard de cette entrée dans la discipline par l'objet, propre aux années 20, les Essais, Prolégomènes, Problèmes, Introductions... des années structuralistes expriment un bougé dans la conscience de soi de la discipline. Il faudrait bien entendu affiner l'analyse et chercher en particulier à différencier cette seconde série d'intitulés les uns par rapport aux autres: les « prolégomènes » « annoncent » dans l'ordre de la légitimation et des fondements, les « essais » mettent à l'épreuve et soumettent au jugement, les «introductions » ménagent des accès, etc... On ne peut toutefois s'empêcher de penser que ce qui a changé relève de la manière dont les théoriciens situent le rapport à l'objet après Saussure. Si l'on admet toutefois que « après Saussure " signifie à peu près ici : "après que Saussure fut devenu une borne de mémoire pour la discipline", et quoi qu'on puisse penser du contenu réel de ces filiations.

\section{1. « Problèmes... » et discipline}

La question du titre n'est pas futile non plus en ce qui concerne Benveniste et le versant « linguistique générale » de son oeuvre. Le linguiste se donne en tous cas le mal d'une justification dans un très court Avant-propos aux Problèmes de linguistique générale $1^{4}$ : si les articles présentés trouvent place dans un recueil qui porte ce titre, c'est qu'ils participent tous à ce que Benveniste nomme ici une «problématique du langage $»^{5}$.

«Problèmes", "problématique » : s'il y a des styles de pensée, et si il y a un style de pensée proprement benvenistien, c'est bien celui de la «problématisation ». Il s'agit avant tout d'ouvrir des perspectives, de mettre en relation, de définir

\footnotetext{
4 On sait que l'organisation des P. L. G. I est due à l'auteur lui-même, P. 1. G. II reprenant cette organisation alors que Benveniste n'était plus en mesure de travailler.

5 «Les études réunies dans cet ouvrage ont été choisies entre beaucoup d'autres, plus techniques, que l'auteur a publiées au long de ces dernières années. $\mathrm{Si}$ on les a présentées ici sous la dénomination de "problèmes ", c'est qu'elles apportent dans leur ensemble et chacune pour soi une contribution à la grande problématique du langage qui s'énonce dans les différents thèmes traités : on y envisage les relations entre le biologique et le culturel, entre la subjectivité et la socialité, entre le signe et l'objet, entre le symbole et la pensée, et aussi les problèmes de l'analyse intralinguistique. Ceux qui découvrent dans d'autres domaines l'importance du langage verront ainsi comment un linguiste aborde quelques unes des questions qu'ils sont amenés à se poser et ils apercevront peut-être que la configuration du langage détermine tous les systèmes sémiotiques ». Avant-propos, PLG I.
} 


\section{Christian Puech}

des points de vue et pour cela de ne pas se satisfaire des découpages disciplinaires certes éprouvés, mais aussi institués. De ce point de vue, les PLG ne manquent jamais une occasion de remettre en cause l'autosuffisance des savoirs linguistiques qui s'accordent d'emblée la consistance disciplinaire dont ils devraient au contraire apporter la preuve.

Cela n'apparaît jamais mieux que lorsque Benveniste investit des terrains du savoirs qui ne sont pas institutionnellement les siens. Problématiser, par exemple, « la forme et le sens dans le langage » (PLG, 2, p. 215) dans un congrès de philosophie suppose de faire jouer les uns par rapport aux autres une succession de points de vue dans une véritable stratégie générale de " mise en crise » des évidences de pensée et des positions disciplinaires qui y sont associées, ou qui en sont le support, bien au delà des précautions rhétoriques d'usage. On se souvient sûrement de l'introduction de cette intervention, et de l'itinéraire critique qu'elle fait parcourir. On peut le schématiser ainsi :

a) l'énoncé du problème posé est plus philosophique que linguistique

b) c'est en linguiste, pourtant, que l'auteur l'abordera

c) il n'existe cependant aucun savoir linguistique constitué en la matière

c) c'est donc en son nom personnel qu'un linguiste parle ici

d) les notions de forme et de sens seront donc abordées hors de tout présupposé philosophique.

Il me semble qu'on a là, explicité dans sa forme, le schéma d'une problématisation de type benvenistien : mise en regard - ou dos à dos - de deux points de vue (ici, linguistique et philosophique) qui invite à chercher une autre voie, engagement personnel qui donne à l'analyse l'aspect d'une «méditation» comportant des risques, impliquant des enjeux de pensée, passion des commencements véritables enfin, qui écarte - au moins par provision et en en tenant compte - les médiations disciplinaires pour affronter l'objet même.

En plusieurs occasions, Benveniste semble se situer ainsi en ce point où le recours à la discipline comme réservoir de certitudes et d'évidences n'est plus possible. C'est le cas encore, par exemple dans " sémiologie de la langue " (rédigé pour la revue Sémiotica; cf. PLG 2 p. 43) où il s'agit, méditant Saussure, de dégager la problématique complexe des relations entre linguistique et sémiologie. S'il convient alors de reprendre le geste saussurien, ce n'est pas pour le répéter ni le corriger, mais plutôt pour le réinvestir et y retrouver une charge conceptuelle potentielle en partie recouverte par l'héritage qu'il a, paradoxalement, légué. Le style proprement "problématisant » de Benveniste consistera donc à reconstruire des principes plutôt qu'à inventorier des résultats déjà évaluables en 1969 dans les travaux de sémiologie ou de sémiotique de l'époque. Il consistera moins à considérer les propositions de Saussure dans le Cours comme la source, la cause première de théorisations ultérieures commensurables entre elles, comparables dans leurs démarches respectives, et susceptibles d'être rapportées à leur point d'origine, que d'en 
retrouver la dimension prospective et projective à travers la construction du concept de langue :

"La préoccupation de Saussure est de découvrir le principe d'unité qui domine la multiplicité des aspects où nous apparaît le langage. Seul ce principe permettra de classer les faits de langage parmi les faits humains [...]

Ici encore on n'a pas assez remarqué la nouveauté de la démarche saussurienne. Il ne s'agit pas de décider si la linguistique est plus proche de la psychologie ou de la sociologie ni de lui trouver une place au sein des disciplines existantes. C'est à un autre niveau que le problème est posé et dans des termes qui créent leurs propres concepts. La linguistique fait partie d'une science qui n'existe pas encore, qui s'occupera des autres systèmes du même ordre dans l'ensemble des faits humains, la SEMIOLOGIE ». [op. cit. p. 47 ; nous soulignons].

La promotion d'une "sémiologie de deuxième génération » ne peut se formuler, selon un paradoxe qui n'est qu'apparent, que par un retour à la dimension projective du geste par lequel le Saussure du Cours institue la sémiologie de première génération : il s'agissait pour le genevois, rappelle ici Benveniste, non de situer la linguistique dans une classification des disciplines de l'homme définitivement acquise, mais de rappeler ces dernières, au contraire, à l'ordre commun qui doit ou peut les régir : $l^{\prime}$ ordre sémiologique ${ }^{6}$. De la même manière, c'est par une certaine désorientation dans l'ensemble des disciplines instituées que la linguistique héritée en partie de Saussure peut espérer penser son statut à venir. Plus précisément, c'est moins en multipliant les relations externes avec les autres disciplines qu'en se régénérant de l'intérieur et en ouvrant une nouvelle dimension de signifiance en son sein même, la dimension du discours, que la célèbre distinction proposée entre sémantique et sémiotique doit permettre une nouvelle distribution des savoirs du signe.

\section{Les partages disciplinaires}

Ce scepticisme vis à vis d'une certaine forme "d'interdisciplinarité », de juxtaposition externe, on le trouve déjà, à l'époque de l'installation du structuralisme triomphant, dans l'article de 1954 sur « Les tendances récentes en linguistique générale " qui ouvre les PLG 1. L'article-bilan, adressé au Journal de Psychologie, pour des "non spécialistes ", donc, situe les tâches de la linguistique d'une part dans une histoire raisonnée qui est celle des conversions de points de vue de la discipline (définition de l'objet, procédures, place de la signification), d'autre part dans un effort d'autonomisation

\footnotetext{
6 Rappelons à ce sujet que dans les Sources Manuscrites éditées par R. Godel, Saussure n'évoque les autres sciences que sur le mode d'une restriction essentielle, puisqu'elle touche à ce qui fait l'ordre commun de leurs préoccupations : « ... du moins toutes celles qui touchent à la valeur ».
} 


\section{Christian Puech}

croissante de la discipline que Benveniste décrit comme un véritable mouvement d'émancipation :

"Le seul énoncé de ces questions montre que le linguiste veut se défaire des appuis ou des attaches qu'il trouve dans des cadres tout faits ou dans des disciplines voisines " (p. 7).

C'est pourquoi, quand la relation aux disciplines voisines est envisagée, $c^{\prime}$ est toujours dans la perspective d'un renouvellement mutuel et d'une fondation commune - celle, le plus souvent, d'une science générale de la culture - elle-même soumise à un préalable indispensable : la réintégration du sens dans l'analyse linguistique, la prise en compte des processus de "symbolisation » dans la psychologie et la sociologie. De ce point de vue, le formalisme américain est doublement coupable. Il est coupable une première fois parce que

"Cette manière de formaliser les parties de l'énoncé risque d'aboutir à une nouvelle atomisation de la langue [...] On peut (donc) concevoir plusieurs types de description et plusieurs types de formalisation, mais toutes doivent nécessairement supposer que leur objet, la langue, est informé de signification, que c'est par là qu'il est structuré, et que cette condition est essentielle au fonctionnement de la langue parmi les autres systèmes de signes » [PLG1, p. 12]

Mais il est coupable une seconde fois puisque :

"Il est à craindre que, si cette méthode doit se généraliser, la linguistique ne puisse rejoindre aucune des autres sciences de l'homme ni de la culture » [ibid]

D'une manière plus générale, les considérations disciplinaires et interdisciplinaires qui ont accompagné le développement de la linguistique structurale selon des modalités variées mais omniprésentes concernent surtout, chez Benveniste, la modalité prospective. Comme chez Saussure, elles concernent davantage la perspective ou le principe d'une science générale de la culture, ou d'une refondation de la sémiologie ${ }^{7}$ conçue comme horizon, que l'analyse linguistique actuelle, ou le souci d'assigner un statut à la linguistique dans le champ des sciences humaines instituées.

On ne trouvera pas chez lui, par exemple, l'appréhension des sciences de l'homme à visée encyclopédique qu'on trouve chez l'autre phare de la linguistique contemporaine, R. Jakobson, pour qui la juxtaposition systématique, la totalisation, la configuration homogène des savoirs est une tentation constante. Il suffit ici de rappeler la démarche du Rapport rédigé pour l'UNESCO sur «les relations entre la science du langage et les autres sciences $»^{8}$.

7 Sur la sémiologie de Benveniste comme «point de fuite» de l'analyse linguistique, cf. C. Normand, 1989; 1992 pour le traitement de la signification, 1986 sur la conception de l'énonciation, 1985 pour une mise en perspective de la problématique de l'énonciation.

8 (1970) in Essais de linguistique générale **, pp. 9-113, Les Editions de Minuit, 1979, Paris. 
On se souvient que Jakobson y répartit les sciences humaines instituées sur trois cercles concentriques :

"Trois sciences appartiennent à un ensemble s'englobant l'une l'autre et représentant trois degrés de généralisation croissante: 1) l'étude de la communication de messages verbaux, ou linguistiques, 2) l'étude de la communication de messages quelconques, ou sémiotique (y compris la communication de messages verbaux), 3) l'étude de la communication ou anthroplogie sociale et économique (y compris la communication de messages) » [op. cit. p. 37]

La fin du Rapport permet sans aucun doute de rajouter un quatrième cercle : celui qui concerne les "sciences biologiques de la communication" (pp.54-55 en particulier). Malgré des préoccupations très proches (qui ressortissent au souci de situer la linguistique dans une anthropologie générale), n'est-on pas ici aux antipodes de la démarche du Benveniste " généraliste »?

- La représentation du savoir dans la clôture d'un cercle,

- l'image d'un emboîtement des domaines dans une perspective à la fois hiérarchique et cumulative (le deuxième "cercle " incluant le premier, par exemple, en le dominant manifestement),

- l'idée même, peut-être, d'un ordre des connaissances procédant par généralisation croissante.

Sans doute le point de vue disciplinaire est-il toujours un point de vue intégrateur : il implique par nature une mise en relation, la représentation du savoir comme unité articulée, la construction d'un champ homogène à partir $\mathrm{d}^{\prime}$ une ontologie au moins implicite. Mais c'est bien parce qu'on a affaire à une construction qu'un point de vue comparatif est possible.

Ainsi, lorsque Benveniste envisage les relations de la linguistique aux autre disciplines, on peut dire au contraire que c'est presque toujours :

- dans un horizon idéal, ou un avenir indéterminé qui vaut comme principe régulateur plus que critère de classement au sens strict (sémiologie, sémiologie de deuxième génération, science générale de la culture),

- moins dans une perspective encyclopédique que fondatrice (quel sort pour la signification en linguistique et dans les autres disciplines de la culture?),

- dans un ordre qui ne va pas du moins au plus général, mais plutôt du simple au complexe, et sans continuité.

- dans une coupure radicale avec les sciences de la nature enfin.

Significativement, il me semble que c'est dans le texte commémoratif sur Saussure ${ }^{9} \mathrm{qu}^{\prime}$ on trouve l'expression la plus claire de cet horizon de projection de la linguistique chez Benveniste. Ici, l'idée d'une science générale de la culture est envisagée essentiellement à partir de la considération des préalables

9 (1963) « Saussure après un demi-siècle », PLG1, p. 32-45. 


\section{Christian Puech}

auxquels elle devrait obligatoirement satisfaire. Tout particulièrement, et vraisemblablement à l'origine même de la vision projective de Benveniste, la distinction entre fait de nature et fait de culture ${ }^{10}$ :

"Il nous semble qu'on devra établir une distinction fondamentale entre deux ordres de phénomènes: d'une part les données physiques et biologiques, qui offrent une nature "simple» (quelle que soit leur complexité) parce qu'elles tiennent entièrement dans le champ où elles se manifestent, et que toutes leurs structures se forment et se diversifient à des niveaux successivement atteints dans l'ordre des mêmes relations; et d'autre part les phénomènes propres au milieu interhumain, qui ont cette caractéristique de ne pouvoir jamais être pris comme données simples ni se définir dans l'ordre de leur propre nature, mais doivent toujours être reçus comme double, du fait qu'ils se relient à autre chose, quelle que soit leur "référent». Un fait de culture n'est tel qu'en tant qu'il renvoie à quelque chose d'autre. Le jour où une science de la culture prendra forme, elle se fondera probablement sur ce fait primordial, elle élaborera ses dualités propres à partir du modèle qu'en a donné Saussure pour la langue, sans s'y conformer nécessairement ». [op. cit. p. 44].

Ici, pas de continuité « englobante » du simple au complexe, mais plutôt un saut, qui est le saut toujours déjà accompli dans la culture. Pas de véritable hiérarchie non plus entre les disciplines mais une convergence de perspective d'ordre formel liée à cette sorte d'ontologie négative ou d'anti-ontologie de la définition saussurienne du signe. Sans se restreindre à cette dimension disciplinaire et prospective, la distinction entre sémiotique et sémantique, l'ouverture de la dimension de la signifiance dans l'analyse de la langue fondatrice de la "sémiologie de deuxième génération » est à la fois cause et effet d'un retour réflexif de la linguistique sur elle même, comme si le geste saussurien d'institution de la sémiologie ne pouvait être dépassé que par un prolongement, car :

"La sémiologie de la langue a été bloquée, paradoxalement, par l'instrument même qui l' a créé : le signe » [op. cit. p.66].

C'est sans doute pourquoi, et pour finir, il est possible de repérer - trop schématiquement - dans les articles les plus « exotériques » de Benveniste certaines modalités de la «mémoire disciplinaire » et de la filiation saussurienne du linguiste.

\footnotetext{
10 On ne peut éviter de mettre en relation cette anthropologie prospective de Benveniste avec les travaux de M. Merleau-Ponty, son collègue au Collège de France. Phenoménologie de la perception (p. 518) : «Tout ce que nous sommes nous le sommes sur la base d'une situation de fait que nous faisons nôtre et que nous transformons sans cesse par une sorte d'échappement qui n'est jamais une liberté inconditionnée ». Signes (p. 115) : «La parole, en tant que distincte de la langue, est ce moment où l'intention de signifier encore muette et tout en acte, s'avère capable de s'incorporer à la culture, la mienne et celle d'autrui, de me former et de le former, en transformant le sens des instruments culturels. Le document sollicite et fait converger toutes les idées connaissantes, et à ce titre instaure et restaure un « logos » du monde culturel »
} 


\section{La structuration de la mémoire disciplinaire.}

Il s'agirait ici essentiellement des cinq articles qui se distribuent dans les deux tomes des Problèmes de linguistiques générale sous l'intitulé « Transformations de la linguistique ».

A) On peut considérer d'abord que ce qui fait leur valeur, du point de vue qui est le nôtre, c'est qu'il s'agit d'écrits de circonstance. La place accordée aux entretiens journalistiques dans le deuxième tome marque bien l'importance des transformations exotériques du statut de la linguistique des années cinquante à la fin des années soixante : d'expert qu'il était auprès d'experts de disciplines voisines ${ }^{11}$, le linguiste est devenu - pour un temps au moins - le détenteur supposé d'un sens - un «maitre» en réalité ironique et amusé auquel on s'adresse pour éclairer une configuration culturelle qui intéresse «le plus grand nombre $»$.

B) L'histoire pratiquée par Benveniste dans ces cinq articles n'est jamais une histoire "saturée": elle ne vise jamais - même quand il s'agit de commémorer Saussure - à déterminer une origine absolue ou un objectif ultime, elle fait peu de place aux notions historiographiques d'école ou d'influence, mais s'attache plutôt à la diversité, aux variétés de l'objet, comme si l'histoire était pour lui la continuation par d'autres moyens de l'épistémologie des principes mise en évidence tout à l'heure à propos des démarquages disciplinaires. De ce point de vue, la conclusion de «Tendances récentes en linguistique générale » (1954) est emblématique :

«Si particulière sont les conditions propres au langage qu'on peut poser en fait qu'il n'y a pas une mais plusieurs structures de la langue, dont chacune donnerait lieu à une linguistique complète » [PLG 1, p. 16]

Ici, la diversité dans l'ordre des successions et des théories est finalement convertie en variété et ouverture synchronique, occasion d'un retour réflexif sur l'objet et sur les principes.

C) C'est que l'histoire pratiquée par Benveniste est une histoire problématisée qui ne saurait se satisfaire d'une chronique ou d'un inventaire d'ordre quantitatif, en effet :

"... la multiplication des travaux ne révèle pas immédiatement, mais masque plutôt les transformations profondes que subissent la méthode et l'esprit de la linguistique depuis quelques décennies et les conflits qui la divisent aujourd'hui » [ibid. p. 4]

A ce titre, c'est aussi une histoire animée d'un mouvement paradoxal . Si d'un côté, selon Benveniste, la linguistique historique et comparée du XIX ${ }^{\circ}$ et début $\mathrm{du} X \mathrm{X}^{\circ}$ siècle reste figée dans «la successivité comme principe

\footnotetext{
11 L'article destiné au Journal de psychologie s'inscrit en fait dans une véritable série depuis le début du siècle. On devrait pouvoir suivre les constantes et transformations de la «présentation de soi » de la linguistique à travers les différents numéros spéciaux des revues de psychologie consacrés au langage.
} 


\section{Christian Puech}

d'explication», "le morcellement en éléments isolés", "une conception évolutionniste de la loi qui régit les faits linguistiques ", d'un autre côté, la linguistique générale qui rompt avec elle - celle promue par Saussure - n’en est que l'aboutissement ultime :

"Tout l'effort de Saussure [...] c'est l' exigence qu'il a posée d'apprendre au linguiste ce qu'il fait ».

La référence à Saussure dans l'horizon de rétrospection de Benveniste est ainsi moins historique, au sens où quelque chose aurait commencé avec lui qui n'aurait eu qu'à se poursuivre, à s'amender, à se compléter selon une cumulativité progressive, qu'elle n'est fondatrice dans l'ordre de la légitimité :

"Saussure, ce n'est pas un commencement, c'est autre chose, ou c'est un autre type de commencement » [PLG II, p. 16]

Commencement, l'oeuvre de Saussure est commencement «dans un autre ordre ", donc. S'il n'est pas question ici de relever de manière exhaustive les différentes modalités de la référence à Saussure ${ }^{12}$ chez le linguiste des Problèmes, il semble bien toutefois que le linguiste genevois y figure moins, en général, sous les traits du prédécesseur que sous ceux de l'interlocuteur qu'il est d'ailleurs parfois possible d'opposer à lui-même.

D) On pourrait comparer là encore Benveniste et le Jakobson de « La théorie saussurienne en rétrospection» (Selected Writings VIII ; pp 393-435 S. Rudy ed., Mouton). Dans ce dernier texte, Jakobson recourt au détour historique pour préciser la spécificité de sa propre démarche. Le Cours, y explique-t-il,

«... au lieu d'un édifice accompli...ne représente que le commencement mouvementé d'une édification nouvelle et mouvementée » [p. 396, je souligne]

Complexité des commencements! Ce qui n'est pas un commencement pour Benveniste, qui est, en tous cas, un commencement "d'un autre ordre ", un recommencement, n'est donc qu'un commencement pour le linguiste pragois. La restriction n'introduit pas ici une dévalorisation des spéculations du Cours, mais bien une autre valorisation : Saussure est un prédécesseur, ses propositions théoriques des « essais » dont la cohérence est, pour de multiples raisons, fragiles. Elles s'inscrivent dans une série empirique de théorisations et appellent des prolongements conformément aux deux sens du mot «essai » : à la fois tentative inachevée et mise à l'épreuve ${ }^{13}$. Ce n'est plus en revenant au geste saussurien, même pour le dépasser ou l'ouvrir à de nouvelles

12 cf. Les différents articles de C. Normand signalés plus haut.

13 Jakobson répartit souvent les grandes oeuvres linguistiques du passé en deux grandes catégories : certaines, à l'image des Prinzipien d'H. Paul, sont des oeuvres achevées, des synthèses, des «épilogues ». D'autres, dont le CLG sont des «prologues »: "Saussure porte son attention sur ce qui reste à faire... » cf. "La théorie saussurienne en rétrospection», op. cit., mais aussi "Zur Struktur des Phonem » S. W. I, 1939. 
potentialités, qu'on introduira dans l'analyse linguistique la dimension du dialogue, celle de l'intersubjectivité, des interactions. Ce dont il s'agit, c'est plutôt d'un inventaire des manques constitutifs de son entreprise, qui exige la mise en oeuvre d'une épistémologie des erreurs et des lacunes, plutôt qu'une épistémologie des principes et des potentialités. Par exemple :

"Saussure fait malheureusement abstraction du rôle de l'auditeur » [op. cit., p. 404]

ou bien encore

«Nous aboutissons nécessairement à une modification de la doctrine saussurienne concernant la langue et la parole. La langue a, à côté de l'aspect social, un aspect individuel et la parole a, à côté de l'aspect individuel, un aspect social » [op. cit. p. 414]

Pour conclure ces remarques schématiques, on fera remarquer que la perspective choisie ici laisse délibérément de côté - où se tient tout juste en deçà - des questions capitales: qu'est ce qu'un problème de linguistique générale pour Benveniste? Le style "problématisant » de Benveniste n'est-il qu'un mode d'exposition? Voire même un mode d'exposition qui homogénéise dans son style des analyses descriptives de la langue et des propositions intellectuelles au statut plus incertain dans une stratégie de fuite en avant, dont la sémiologie générale ne constitue qu'un horizon asymptotique ?

Quoiqu'il en soit, tant le choix d'une conceptualisation par approfondissement, tant la vigilance critique exercée vis à vis de toute démarche externe dans la délimitation des objets de connaissance de la linguistique et dans la perspective d'une science générale de la culture, attirent bien l'attention sur la diversité des modes par lesquels les propositions les plus innovantes des linguistes s'insèrent dans une représentation de la discipline qu'ils construisent souvent à partir des mêmes références.

De ce point de vue, la question est peut-être moins de savoir dans quelle mesure la linguistique structuraliste procède bien de l'enseignement saussurien, que de mesurer jusqu'à quel point et selon quelles modalités variées le Cours a bien libéré chez certains linguistes, une dynamique de questions ouvertes et risquées qui doit d'abord se construire une mémoire et reconstruire sans cesse sa source la plus vive.

En ce qui concerne Benveniste, qui ne manque jamais dans ses entretiens des années 68/70 de noter le comique de la fétichisation tardive du Cours, l'oeuvre ouverte de Saussure prophétise sans doute moins un structuralisme de méthode et de doctrine voué à la généralisation, qu'elle n'annonce de manière intempestive, dans ses principes mêmes, son dépassement. L'oeuvre saussurienne n'est-elle pas constituée de plusieurs

"[...] ensembles d'aperçus généraux, dont chacun appelle une exégèse et dont certains nourrissent encore la controverse, projetant les langues sur le plan d'une sémiologie 
Christian Puech

universelle, ouvrant des vues auxquelles la pensée philosophique d'aujourd'hui s'éveille à peine » [PLG I, p. 7]

Dans cette mesure même, Benveniste indique bien à quel point faire l'histoire du structuralisme linguistique impliquerait de se rendre attentif en même temps à la diversité des modes de disciplinarisation de propositions théoriques qui ne se laissent pas enfermer dans une chronologie linéaire, et à la diversité des références à un texte fondateur qu'il s'agit moins de découvrir que « $d$ 'inventer » à chaque fois.

\section{Bibliographie}

BENVENISTE, E. (1966 - 1974) : Problèmes de linguistique générale I et II, Paris, cité dans l'édition TEL, Gallimard.

BLOOMFIELD, L. (1927) : « On recent Work in General Linguistics », in (1970) A Leonard Bloomfield Anthologie, C. F. Hockett ed., Bloomington \& London : Indiana University Press.

CHISS, J. L. et Puech, C. (1994) : «Saussure et la constitution d'un domaine de mémoire pour la linguistique moderne ", Langages 114, Paris, Larousse.

CHISS, J. L. Et Puech, C. (1995) : «La linguistique structurale du discours de fondation à l'émergence disciplinaire ", Langages 120, Paris, Larousse.

JAKOBSON, R. (1973) Essais de linguistique générale 2, Paris, Les éditions de Minuit.

JAKOBSON, R. (1985) "La théorie saussurienne en rétrospection» Roman Jakobson Selected Writings, S. Rudy ed.; vol. VIII, Berlin, Nex york, Amsterdam: Mouton Publishers.

NORMAND, C. (1986): «Les termes de l'énonciation de Benveniste»; Histoire, Epistémologie, Langage, t. 8 - fasc. II.

NORMAND, C. (1989) : «Constitution de la sémiologie chez Benveniste», Histoire, Epistémologie, Langage t. 11, fasc. II

NORMAND, C. (1992): «Benveniste: linguistique saussurienne et signification», LINX 26, Université Paris X Nanterre.

PUECH, C. (ed.) (1992) : «Sémiologie et histoire des théories du langage », Langages 107, Paris, Larousse.

SAPIR, E. (1953) : Le langage, Paris, Payot

SAPIR, E. (1968) : « La place de la linguistique parmi les sciences », Linguistique, Paris, Les Editions de Minuit.

VENDRYES, J. (1920) : Le langage : une introduction à l'histoire, Paris, Albin Michel 